\title{
PRODUÇÃO CIENTÍFICA SOBRE PLÁGIO INDEXADA NA LISA
}

\author{
Ednéia Silva Santos Rocha* \\ Márcia Regina da Silva*
}

RESUMO

\begin{abstract}
Neste trabalho propomos refletir sobre a produção científica internacional relacionada ao tema plágio na área de Ciência da Informação. Trata-se de uma pesquisa quantitativa e do tipo exploratória que analisou artigos científicos da área de Ciência da Informação indexados na base de dados Library and Information Science Abstracts (LISA). O corpus de análise foi composto por 35I artigos científicos sobre a temática. Como resultado destaca-se que há concentração de artigos sobre plágio a partir da década de 2000; os principais temas abordados nas publicações se referem ao copyright; a palavrachave plágio está associada principalmente com os temas 'ethic', 'detection', 'user training' e 'information literacy'. O periódico de maior destaque é o Journal of Information Ethics (JTE) e o idioma predominante é o inglês. A produção científica internacional da Ciência da Informação sobre plágio volta-se para diversas questões, destacando-se estudos que atribuem ao bibliotecário e educadores a responsabilidade na formação de estudantes comprometidos com a integridade científica. É importante que no Brasil também haja essa preocupação na produção científica da área de Ciência da Informação.
\end{abstract}

Palavras-chave: Indicadores Bibliométricos. Plágio. Ciência da Informação.

\footnotetext{
* Doutora em Política Científica e Tecnológica pela Universidade Estadual de Campinas, Brasil. Bibliotecária da Faculdade de Direito de Ribeirão Preto da Universidade de São Paulo, Brasil. E-mail: edneia@usp.br.

* Doutora em Educação pela Universidade Federal de São Carlos, Brasil. Coordenadora do Curso de Biblioteconomia, Ciências da Informação e da Documentação da Faculdade de Educação, Ciências e Letras de Ribeirão Preto Universidade de São Paulo, Brasil. E-mail: marciaregina@usp.br.
}

\section{INTRODUÇÃO}

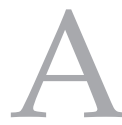

construção de um texto científico requer o levantamento criterioso de informações científicas em fontes confiáveis. Uma das características da sociedade atual é a possibilidade de acessar informações disponibilizadas em fontes de informação de natureza diversa que circulam pela rede. As bibliotecas digitais e os repositórios, por exemplo, tornaram-se ferramentas primordiais para a construção do conhecimento. Obras antes restritas a um público específico, hoje são disponibilizadas em formato digital, podendo ser acessadas de qualquer ponto de rede.

Da mesma forma que hoje há maior disponibilidade de informações no ambiente digital, há também maior possibilidade de ocorrer o plágio. O plágio pode ser definido como a apropriação de ideias e/obras alheias sem a devida citação. No Brasil, a Lei n. 9610/1998 trata dos direitos autorais e considera falsificação a reprodução não autorizada de uma obra. Embora não seja uma Lei específica para o plágio, tratase de um crime contra a Propriedade Intelectual, sendo previsto pena de detenção.

Segundo Cameron, Zhao e McHugh (2012) a discussão sobre o plágio geralmente envolve três questões: 1) o plágio como infração notória, geralmente as palavras 'crime' e 'roubo' aparecem associadas a essa prática; 2) a ausência de intenção de plagiar não é um fator atenuante; e 3) erros, mesmo que triviais, na forma de citação ou confusões no texto podem ser interpretados como plágio. Essas questões demonstram a importância de reflexões nesse âmbito como forma de contribuir para a integridade científica. 
O plágio é considerado uma prática desonesta que infelizmente está presente no meio acadêmico e científico das diversas áreas do conhecimento. Na área de saúde, Pádua e Guilhem (2015) corroboram com essa afirmação. Por meio de uma revisão sistemática, os autores concluíram que a literatura sobre a integridade científica ainda é incipiente no Brasil, no entanto, tem um delineamento maior para a questão do plágio e conflitos de interesse de autoria. Embora a detecção do plágio tenha se tornado mais dinâmico no meio acadêmico, com o aparecimento de ferramentas aplicadas ao ambiente digital, que conseguem identificar tal prática, os estudantes ainda demonstram ter pouco conhecimento a respeito; é o que foi identificado do trabalho de Guedes e Gomes Filho (2015) que avaliaram o nível de conhecimento dos alunos matriculados no curso de odontologia da Universidade Estadual do Sudoeste da Bahia. Apesar de utilizarem fontes confiáveis para as pesquisas, os alunos não apresentaram conhecimento pleno sobre o plágio e mostraram não respeitar os direitos autorais das imagens.

O profissional formado na área de Ciência da Informação tem como habilidade a capacidade de manipular fontes de informação diversas em formato manual ou eletrônico, tem acesso competente aos repositórios, bases de dados, bibliotecas digitais, catálogos on-line, etc. Tal competência em informação pode contribuir para a seleção de fontes confiáveis, o que infere a acuidade na construção do conhecimento científico. Sob outra perspectiva, o acesso facilitado a repertórios diversos para a busca de informações pode contribuir para o aumento dos casos de plágio. Esse é um problema geral identificado nas diversas áreas do conhecimento, mas pouco retratado no âmbito da produção científica nacional da Ciência da Informação.

Neste trabalho propomos refletir sobre a produção científica internacional relacionada ao tema plágio na área de Ciência da Informação. Para tal, será utilizada como fonte de pesquisa a base de dados Library and Information Science Abstracts - LISA. A escolha de uma base internacional tem justamente a intenção de delinear a questão do plágio sob uma perspectiva externa, contribuindo para discussões com essa vertente na Ciência da Informação brasileira.

Será utilizada como abordagem metodológica a Bibliometria que contribuirá para a construção de indicadores sobre o plágio na produção científica internacional da CI, além disso, a partir da análise das publicações será possível observar o posicionamento da comunidade científica internacional da área da CI frente às questões relacionadas a integridade no desenvolvimento da atividade científica, que envolvem à ética e ciência e a postura do pesquisador.

\section{PROCEDIMENTOS METODOLÓGICOS}

A aplicação de estudos métricos é uma forma reconhecida para a construção de indicadores de ciência e tecnologia (C\&T), sendo freqüentemente empregados na formulação e avaliação de políticas para o desenvolvimento científico e tecnológico. Silva e Hayashi (2011) ressaltam que o interesse por esses estudos está sustentado, principalmente, na necessidade das áreas do conhecimento em mensurar, mapear e caracterizar a produtividade científica seja de uma disciplina específica, de um pesquisador isolado ou um grupo de pesquisadores, de uma coleção de artigos ou de uma instituição. Existem uma variedade de formas, ferramentas e métodos para a construção de indicadores de produção científica, sendo a Bibliometria uma das mais utilizadas.

As informações produzidas e publicadas nos tipos da comunicação científica, especialmente os formais, compõem a chamada literatura científica (STORER, 1966) ou técnica-científica (LE COADIC, 1996). Targino (2000, p. 65) acrescenta que: "[...] ao registrar seus resultados, o pesquisador tem em mente o possível veículo de divulgação, a fim de adaptar o seu documento as exigências das normas editoriais e ao público". A Bibliometria está relacionada à ideia de representar o conhecimento humano por meio da quantificação de documentos, mais especificamente da literatura científica produzida (JIMÉNEZ-CONTRERAS, 2000).

Os estudos bibliométricos oferecem um indicativo do nível de conhecimentos que vem sendo produzido pelos pesquisadores, assim como os temas mais abordados na variedade de aspectos embutidos dentro do tema em questão, permitindo a visualização do cenário e de como os cientistas da informação se inserem nesse contexto, a partir de sua produção bibliográfica, bem como os principais veículos nos quais eles publicam. 
De acordo com Durieux e Gevenois (2010), os indicadores bibliométricos são importantes para os pesquisadores porque permitem medições objetivas da difusão e do impacto dos artigos publicados em uma comunidade científica. Estes indicadores podem ajudar os pesquisadores a selecionar as revistas às quais pretendem submeter suas pesquisas. Para as organizações, indicadores bibliométricos são importantes porque permitem que medidas objetivas sobre a qualidade de uma pesquisa particular, sobre pesquisadores individuais, ou um grupo pesquisadores. Na prática, estes indicadores podem ajudar as organizações a tomar decisões sobre nomeações, promoções e financiamento.

Os indicadores bibliométricos podem também serem usados para determinar a origem geográfica de investigação e detectar o crescimento ou a extinção de temas científicos de acordo com o país de origem (RAHMAN; FUKUI, 2002). Esses indicadores podem ser usados para medir o foco de investigação e detectar a má alocação de recursos de pesquisa. Eles também podem ser usados para prever o sucesso de investigação e para estudar o financiamento de pesquisa, otimizando as políticas de ciência e tecnologia (DURIEUX; GEVENOIS, 2010).

\section{I Descritivo sobre a elaboração dos indicadores bibliométricos sobre plágio}

Essa pesquisa contemplou a construção de indicadores bibliométricos das publicações indexadas na Library and Information Science Abstracts (LISA). A LISA é uma ferramenta internacional de resumos e indexação, elaborada para profissionais da área de Biblioteconomia e outros especialistas da área da informação. A base de dados conta atualmente com resumos de mais de 440 periódicos, publicados em mais de 68 países, num número superior a 20 idiomas. A freqüência de atualização é quinzenal, com mais de 500 registros adicionados em cada atualização. Os principais assuntos indexados são: Gerenciamento da informação; Tecnologia da informação; Tecnologia da Internet; Gerenciamento do conhecimento; Biblioteconomia; Bibliotecas e arquivos; Gerenciamento bibliotecário; Tecnologia bibliotecária; Uso da biblioteca e usuários; Recuperação de informações online.

A fonte de dados para a extração do corpus de análise foi composta por 351 artigos científicos sobre o tema plágio. A Figura 1 traz os detalhes sobre as etapas da pesquisa.

Figura 1: Procedimentos metodológicos

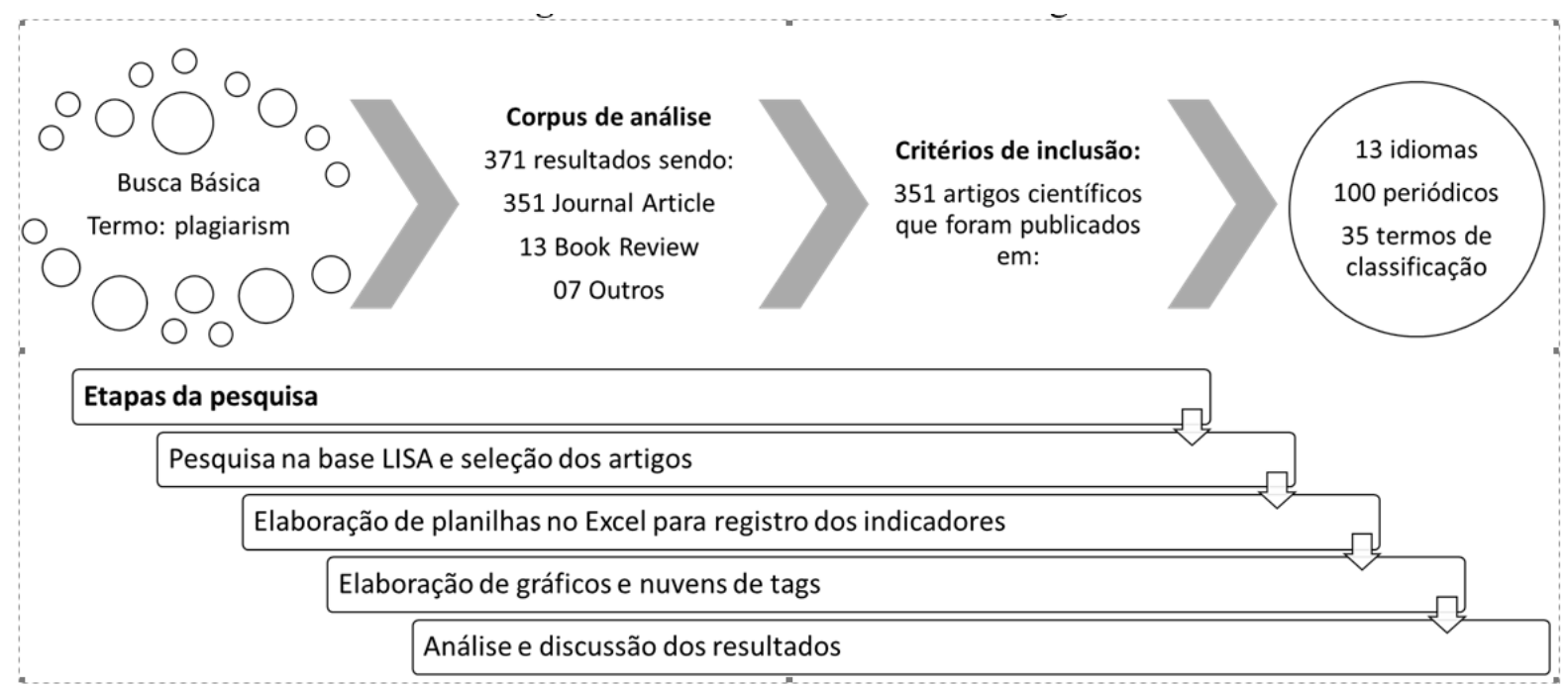

Fonte: Elaboração própria 
Como estratégia de busca foi utilizada o termo 'plagiarism' no campo de busca simples da LISA. No processo de ordenamento e estruturação dos resultados de todas as publicações sobre o tema plágio, foi estruturada uma base de dados no Microsoft Excel para identificação de tendências ao analisar os indicadores. Os resumos de todos os artigos foram lidos para a apresentação das abordagens dos artigos em relação ao tema plágio.

\section{RESULTADOS}

Os indicadores bibliométricos contemplados nessa pesquisa se referem aos anos de publicação, temáticas abordadas, idioma de publicação e periódicos.

No que se refere ao período das publicações (Gráfico 1), verificou-se que o primeiro artigo indexado na base de dados com esse tema foi na década de 1970. Entre as décadas de 1980 e 1990, somando-se o período, obteve-se 29 artigos. Entre os anos de 2000 e 2009 houve um aumento significativo no total de publicações, perfazendo 168 artigos. A partir de 2010-2015 foram publicados 153 artigos, considerando que a década não está encerrada, a publicação de artigos com essa temática, indexados na LISA, têm aumentado exponencialmente.

Gráfico 1: Publicações por décadas

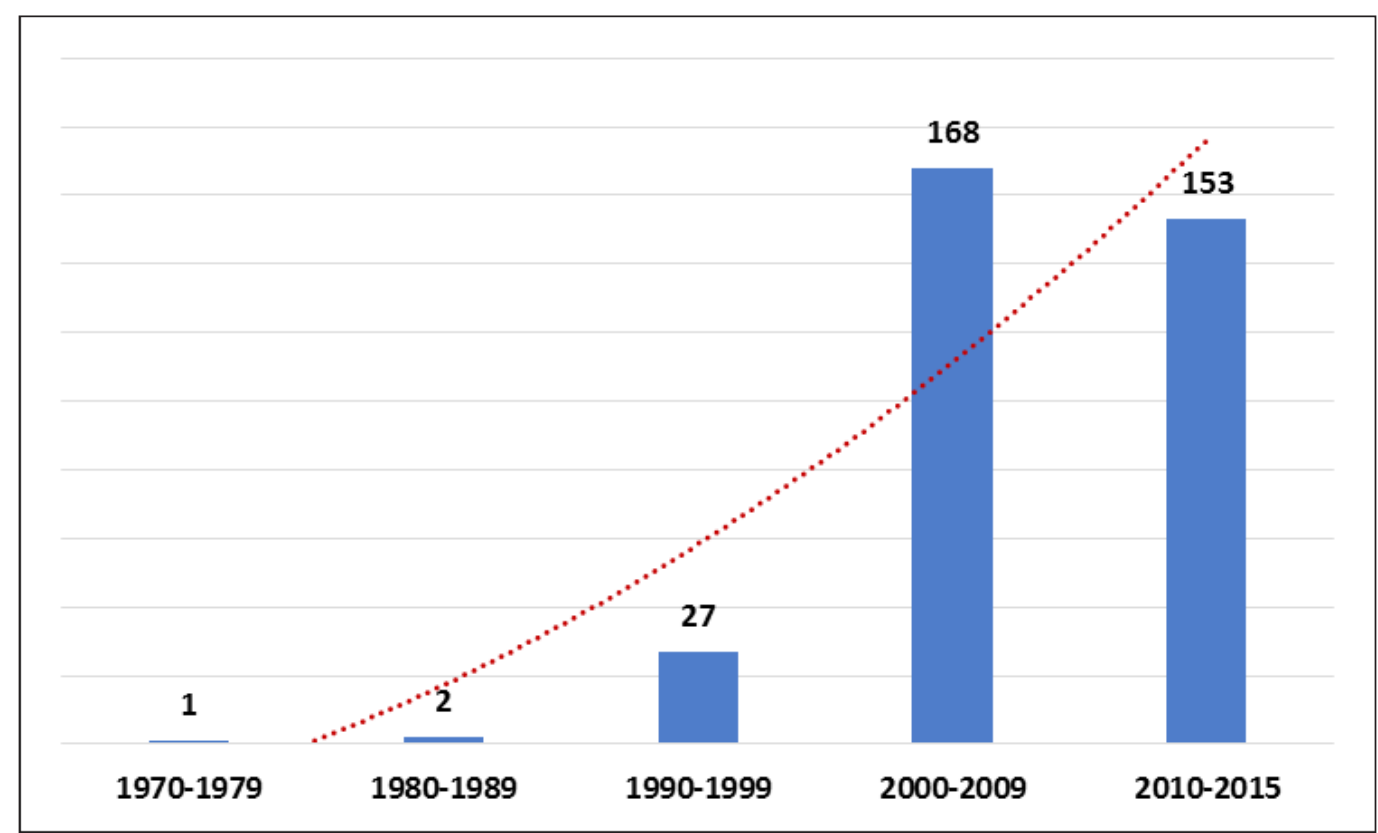

Fonte: Elaboração própria

Desse modo, entre 1970-1979 apenas um trabalho referiu-se ao tema plágio, no entanto, o artigo trata basicamente sobre os direitos autorais, sugerindo o emprego de um sistema de pagamento de taxas para os autores de acordo com as vendas ou um sistema de participação nos lucros das editoras (WALTER, 1976).

No período de 1980-1989 foram recuperadas duas publicações. $\mathrm{O}$ primeiro artigo abordou a responsabilidade dos autores na elaboração de pesquisas com qualidade no Campo da Biblioteconomia. Os autores levantaram casos de plágio, uso de terminologias complexas e falta de detalhamento metodológico nas pesquisas (SEPULVEDA; SEPULVEDA, O., 1981). O segundo artigo verificou casos de plágio e venda de teses em um departamento de história moderna (PISTELLI, 1987). 
O número incipiente de artigos nessas décadas pode relacionar-se a visão de Santos (2011), de que até meados dos anos de 1980, a comunidade acadêmica e os financiadores da pesquisa não possuíam grandes preocupações em torno das questões de integridade da pesquisa. Até esse período, predominava a confiança no respeito dos cientistas à integridade do ethos, de modo que os casos de má conduta acadêmica seriam acontecimentos excepcionais, não justificando a preocupação com ações e políticas sistemáticas que promovessem as práticas éticas na pesquisa (SANTOS, 2011). Os cientistas depositavam grande confiança na capacidade dos debates rotineiros em torno dessas questões e, sobretudo, acreditavam que os mecanismos de revisão por pares seriam suficientes para identificar e coibir as más condutas científicas (SANTOS, 2011).

No período de 1990-1991 foram recuperadas 27 publicações. Entre os temas que se destacam, podemos citar artigos sobre casos de plágio em pesquisas financiadas pelos governos locais (PRICE, 1996; ANDERSON, 1994), sobre a responsabilidade dos editores e revisores nos casos de plágio (RIGGAN, 1997), sobre as controvérsias conceituais sobre o termo plágio (KOZAK, 1994), e sobre casos de plágio em universidades americanas (HANNAY, 1998). Gresham (1996) realizou um estudo sobre a prevenção de plágio na Internet, no qual ressalta a importância da biblioteca ensinar os pesquisadores a forma correta de citar as fontes de informação eletrônica. Já Rees (1997) e Stebelman (1998) abordaram aspectos legais de acesso à informação eletrônica e os problemas relacionados ao uso de documentos eletrônicos sem critérios éticos de pesquisa.

Entre 2000 e 2009 foram recuperados 168 trabalhos, ou seja, $75 \%$ mais trabalhos em comparação a década anterior. Os artigos focaram, principalmente, em temáticas relacionadas a educação e prevenção do plágio (DUGGAN, 2002, 2004; PHILLIPS, 2002); no papel da biblioteca e do bibliotecário na disseminação de uma cultura de integridade científica (BRINE, 2003; MUNDAVA; CHAUDHURI, 2007); nas ações para detecção de plágio (HILL, 2009; HAMILTON, 2003; MADRAY, 2008; BDAKE, 2007); na compreensão do conceito de plágio e direitos autorais (REYMAN, 2008; DAMES, 2007) e; na influência das Tecnologias de Informação e Comunicação (TIC's) nos casos de plágios, com destaque para discussão sobre cyberplágio ou plágio na era digital (BOMBARO, 2007; OLIPHANT, 2002; ERCEGOVAC, RICHARDSON, 2004; AUER, KRUPAR, 2001).

A partir de 2010 até 28 de junho de 2015 (data da coleta de dados), foram publicados 153 artigos. Esses trataram a questão do plágio com abordagens complementares aos estudos anteriores. Por exemplo, Gilson e ChesterFangman (2011) verificaram qual o papel institucional dos bibliotecários na prevenção de casos de plágio e as colaborações entre bibliotecários e professores nas orientações dos estudantes para entender o que é o plágio e como evitá-lo. Ainda analisaram as interações entre bibliotecários e estudantes envolvidos em programas de combate ao plágio. Zimerman (2012) também focou na atuação dos bibliotecários na prevenção e orientação de acadêmicos, enfocando principalmente a interação com estudantes estrangeiros, pois a barreira do idioma pode agravar o problema do plágio entre estudantes oriundos de outros países.

Gipp, Meuschke e Breitinger (2014) levantaram questões relacionadas a detecção de plágio automatizado. Para os autores, tais ferramentas dependem da alta similaridade baseada em caracteres, pois existem formas de plágio fortemente disfarçados, como paráfrases, plágio traduzido, ideias ou estruturas redigidas diferentes da forma apresentada pelo autor que está sendo plagiado, essas ações podem passar despercebidas pelos sistemas automatizados. Como alternativa a esse problema, apresentam a Citation-based Plagiarism Detection (CbPD) que permite a detecção de semelhança semântica, mesmo na ausência de sobreposição de texto, analisando o posicionamento citação no texto completo de um documento para determinar similaridade.

Habibzadeh e Marcovitch (2011) destacam que entre as muitas razões para cometer o plágio, a "preguiça" pode ser a causa mais comum entre os falantes nativos de inglês, mas autores que não dominam o idioma inglês podem reutilizar textos publicados anteriormente porque são relutantes em sacrificar a precisão e qualidade por falta de conhecimentos linguísticos. Barron-Cedeno, Gupta e Rosso (2013) também apresentam a 
relação entre casos de plágio relacionados as traduções e justificam a utilização da CrossLanguage Plagiarism Detection, pois trata da identificação automática e extração de plágio em um ambiente multilíngue.

No Gráfico 2 observa-se que a palavrachave plágio está associada com mais frequência aos temas 'ethic', 'detection', 'user training' e 'information literacy'. Dos 351 artigos, 21\% retratam o tema plágio ressaltando os aspectos éticos, 15\% ressaltam as ações sobre detecção de plágio, 15\% reforçam a ideia sobre o treinamento de usuários para evitar o plágio e $10 \%$ abordam a questão da competência informacional, entre outros.

Gráfico 2: Associação entre termos que possuem relação com plágio

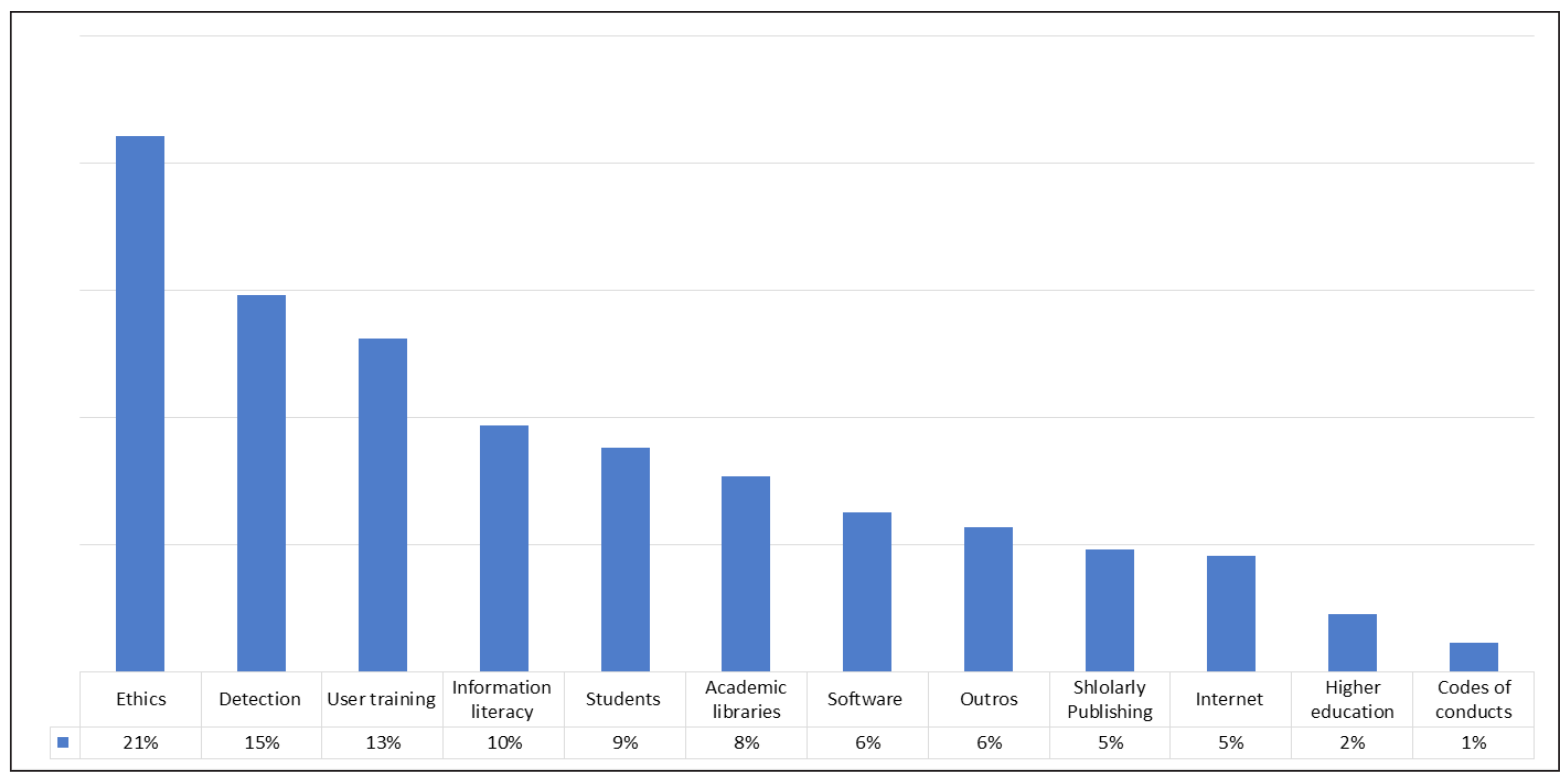

Fonte: Elaboração própria

Em relação a associação do Plágio com a information literacy, ressalta-se que de acordo com a Association of College and Research Libraries (2000), information literacy é definida como a capacidade de saber quando há uma necessidade de informação, sendo o indivíduo capaz de identificar, localizar, avaliar e, efetivamente, usar essa informação. Trata-se de uma competência chave, necessária para melhorar o desempenho acadêmico, melhorar a produtividade no trabalho e competir de forma eficaz em um mercado mundial em constante evolução. Devendo fazer parte dos direitos e garantias individuais, de modo a promover a inclusão digital e social de todos os cidadãos.

Para Fisher, Mouton e Poccoli (1996, p. 7) "as competências informacionais formam a base para a sobrevivência contínua e o crescimento da biblioteca nas diferentes áreas da informação". Nesse cenário, ao analisar o papel do profissional da informação, Dudziak
(2001) constatou mudanças nas ações do profissional como intermediário da informação, mediador do conhecimento e educador. Como mediador, o bibliotecário ensina o usuário a utilizar determinados recursos e ferramentas informacionais, possibilitando desde a busca até a recuperação dos documentos de interesse. Para Belluzzo (2006, p. 82),

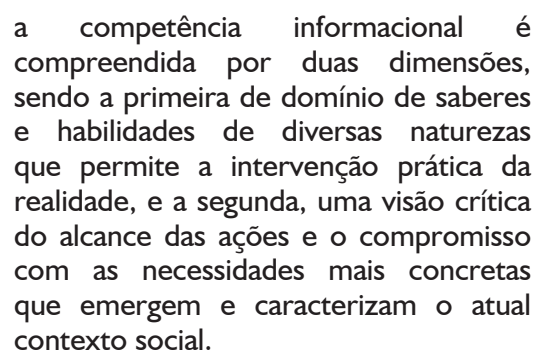

Nessa segunda dimensão, cabe ao profissional da informação estar atento às necessidades dos 
usuários, sendo um facilitador, um agente educacional, de modo a orientar toda a comunidade acadêmica para utilizar corretamente e de maneira ética os recursos informacionais disponíveis. Desse modo, os casos de plágio identificados como negligência em relação ao uso correto das normas de citações e referenciais será minimizado.

A figura 2 demonstra a frequência da incidência dos principais temas das publicações. Uma nuvem de palavras em geral reúne um conjunto de termos utilizadas como palavras chaves das publicações da Base de dados, dispostos em ordem alfabética, e a quantidade de palavras é mostrado proporcionalmente pelo tamanho da fonte. Para essa elaboração dessa nuvem utilizamos Wordclouds (wordclouds.com).

Figura 2: Nuvem de Tags dos termos indexados nas publicações

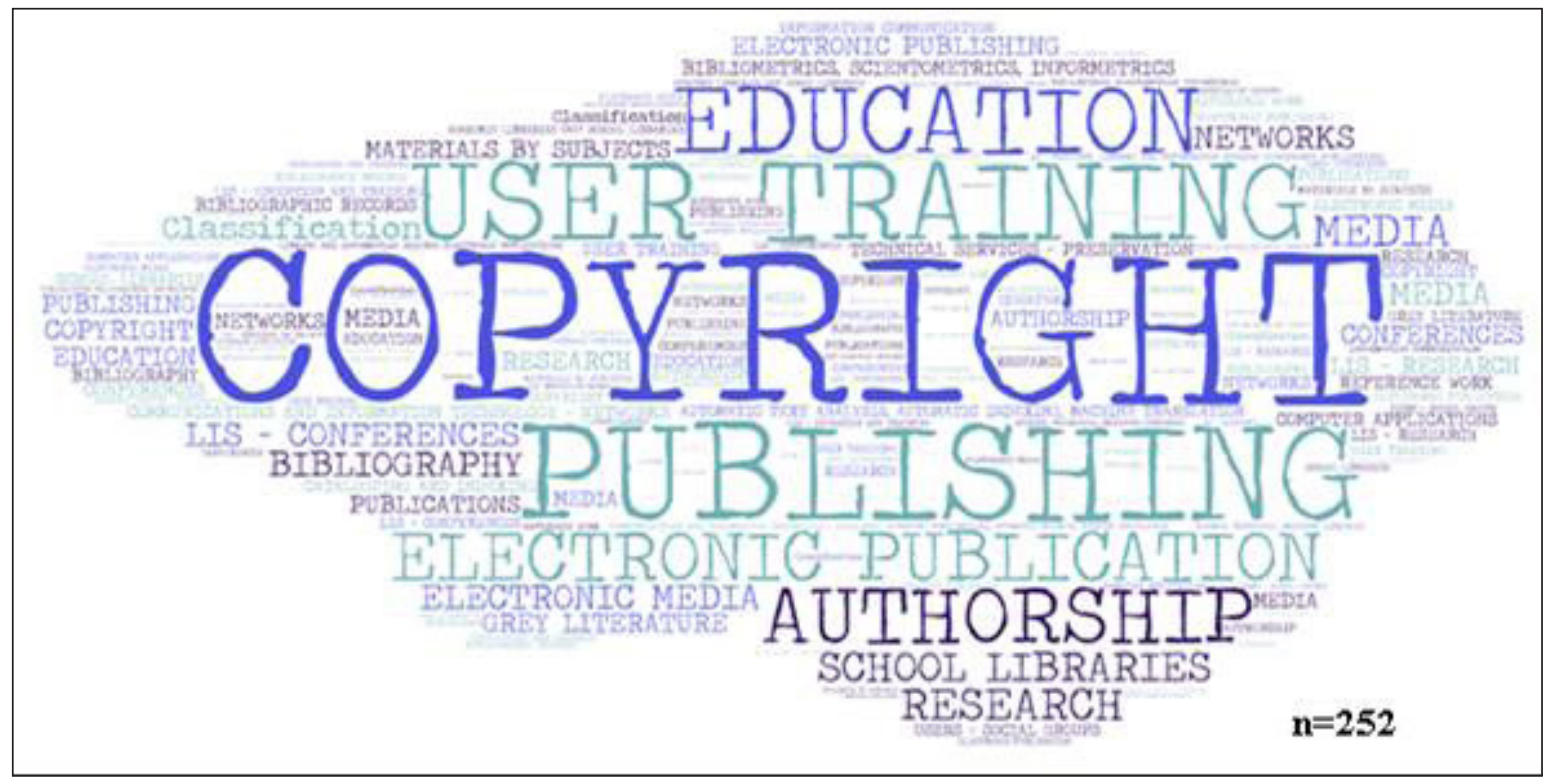

Fonte: Elaboração Própria

Os principais temas abordados nas publicações se referem ao copyright presente em $41 \%$ dos trabalhos. Denomina-se copyright ou direitos de autor, um conjunto de direitos legais que um autor possui sobre a sua produção científica, artística, cultural e intelectual, durante um período de tempo, pois há exceções em relação às obras de 'domínio público', que podem ser reproduzidas e utilizadas por todos. Ou seja, é necessária maior divulgação sobre os direitos autorais, para que haja um maior entendimento da comunidade acadêmica sobre as limitações de se utilizar uma obra que não esteja em domínio público. Embora o copyright não tenha relacionamento direto com o plágio, verificou-se que grande parte dos artigos abrangeu essa questão, isso porque, existindo uma licença de direito autoral, haverá garantia jurídica que nenhuma parte do trabalho poderá ser utilizada sem a permissão do detentor desse direito. Ou seja, no Brasil um suposto plagiador poderá ser responsabilizado juridicamente por crime de Violação aos Direitos Autorais, previsto no Art. 184 do Código Penal.

A temática 'user training', presente em 25\% das publicações, ou seja, em 87 artigos indexadas na LISA, também se destaca. Essa questão sobre treinamento do usuário está diretamente relacionada ao problema da falta de integridade das publicações, principalmente no ambiente acadêmico. Cabem as unidades de informação a tarefa de divulgar a legislação sobre direitos de autor, orientar sobre o uso correto das normas de documentação e informação, explicar o que é considerado má conduta científica, estratégias para evitar o plágio e treinamento dos docentes para utilização das ferramentas tecnológicas de detecção de plágio, para que a comunicação científica tenha alto nível de confiabilidade. 
Os termos 'education', 'electronic publication', 'publishing' e 'authorship' representam juntos 25\% do total de palavras-chave. Sendo que os demais termos apareceram entre uma a três ocorrências. Assim, destaca-se que a educação está associada ao tema plágio, já que as instituições de ensino e pesquisa devem fornecer materiais educativos de combate a má conduta científica para se disseminar no campo educacional uma formação ética. As novas tecnologias, representada pelo termo 'publicação eletrônica', são pertinentes nas discussões sobre plágio, pois com o aumento exponencial das publicações científicas e a facilidade de divulgação das pesquisas no meio digital, o acesso a esses materiais foi facilitado. No entanto, não se deve associar, exclusivamente, o aumento do número de plágios as TICs, pois elas também influenciaram a detecção dos casos de falta de integridade científica. Nota-se a grande disseminação de softwares de detecção de plágio.

Sobre os periódicos científicos, Davarpanah e Aslekia (2008) entendem que essa publicação desempenha papel importante na comunicação científica. Através dos periódicos científicos é possível verificar os problemas que exigem solução, o padrão de pesquisa para solucionar esses problemas, as práticas da atividade científica para as diversas áreas do conhecimento, etc.

Para o levantamento dos principais periódicos que publicaram sobre o tema, foi considerado o percentual acumulado da base de dados, de forma a representar as mais significativas em relação aos resultados apresentados.

Gráfico 3: Principais periódicos que publicaram sobre Plágio na LISA

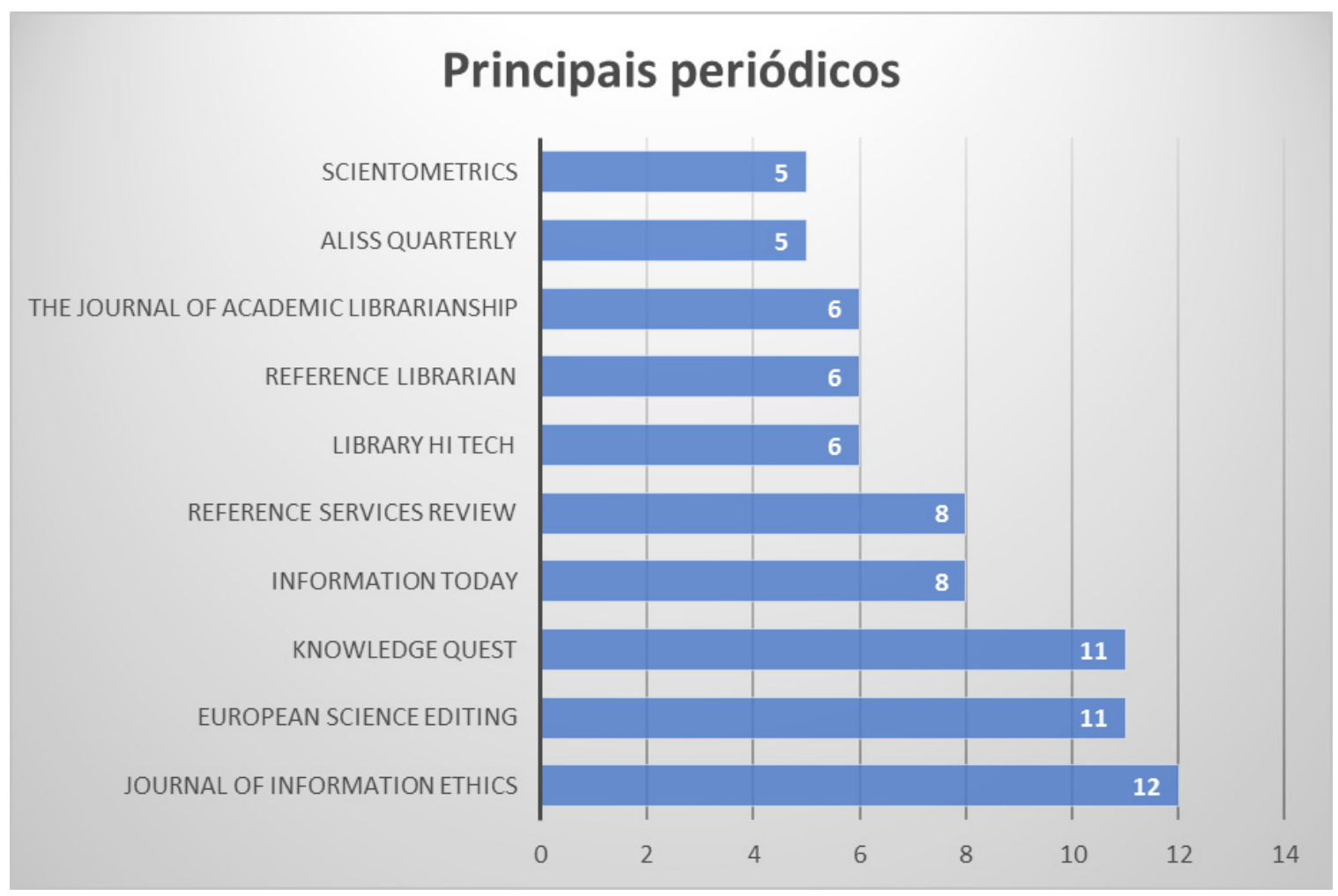

Fonte: Elaboração Própria

Observou-se que há grande dispersão de periódicos, pois os artigos recuperados foram publicados em 100 periódicos diferentes. No entanto, a maioria dos artigos sobre o tema foi publicada no Journal of Information Ethics (JTE) que, possivelmente, é o periódico internacional especifico para tratar de questões éticas relacionadas à informação. De acordo com o 
Diretório periódicos de Ulrich's, esse periódico trata de questões éticas em todas as esferas da Ciência da Informação tendo uma abordagem multidisciplinar.

Entre os artigos publicados no JTE, destacam-se as temáticas: casos de plágio relacionados a ética do conhecimento; ações federais contra plágio em pesquisa; a responsabilidade do revisor/editor em casos de plágio; discussão e definição do conceito de plágio; plágio e cultura universitária; formas de punição àqueles que conscientemente violam a integridade das pesquisas científicas; e serviço de referência em bibliotecas como forma de combater a má conduta cientifica.

Quanto ao idioma, há predominância de artigos publicados no idioma inglês, sendo $90 \%$ dos artigos, tal fato justifica-se pela maioria dos países de língua inglesa nativa produzirem vários trabalhos sobre o tema, outro fator é que a maioria dos periódicos de impactos adotam esse idioma como principal para publicar os artigos.

Cameron, Zhao e McHugh (2012) salientam que a publicação científica mundial tem sido dominada pela língua Inglesa, enquanto a maioria dos cientistas e editoras do mundo não são falantes nativos de inglês. Tal problemática tem implicações importantes para o desenvolvimento da ética em publicações, em particular no que diz respeito ao plágio. Os autores atribuem à falta de compreensão do que se constitui o plágio e o uso de uma estratégia de apoio linguístico, conhecido como patchwriting, à crescente prática de plágio por falantes nãonativos que escrevem em Inglês.

\section{CONCLUSÕES}

Destaca-se a necessidade de se criar uma cultura científica na qual os pesquisadores estejam conscientes da importância de se apresentar dados confiáveis. A prevenção primária é de responsabilidade de todas as comunidades científicas, já que antes da publicação de um artigo, geralmente é realizada a revisão dos pares que devem ter conhecimento da importância de se realizar uma análise bem criteriosa da publicação. As instituições envolvidas com Ciência e Tecnologia devem divulgar as penalidades e sanções que os pesquisadores poderão sofrer em casos em que haja comprovação de fraudes científicas. Um bom exemplo é o "Código de Boas Práticas Científicas", que estabelece diretrizes éticas para as atividades científicas dos pesquisadores beneficiários de auxílios e bolsas da FAPESP e para o exercício da função de avaliador científico pelos assessores da FAPESP. Neste documento há Diretrizes para as atividades científicas, conceituação das más condutas científicas, ressaltando a responsabilidade das instituições de pesquisa e, ainda, descrição os procedimentos relativos à alegação, à investigação e à declaração de más condutas científicas.

No que se refere a questão do plágio na produção científica internacional da Ciência da Informação, observa-se que há estudos que atribuem ao bibliotecário e aos educadores a responsabilidade na formação de estudantes comprometidos com a integridade científica. É importante que no Brasil também haja essa preocupação na produção científica da área de Ciência da Informação.

Por fim, o combate ao plágio passa por uma educação dos jovens pesquisadores ainda na fase inicial de formação - a graduação já que as escolas de ensino médio não estão preocupadas com o rigor científico. Num segundo momento, os avaliadores devem ser mais criteriosos no processo de aprovação de um trabalho científico, cabendo as revistas a divulgação de seus critérios de recusa, dentro os quais o plágio. A questão do plágio extrapola o nível ético das relações científicas, entrando na esfera cível de crime contra os direitos autorais cabendo, no limite, abertura de processo administrativo contra o infrator, podendo levar ao desligamento de suas funções e outras severas penalidades.

A produção científica internacional da área de Ciência da Informação revelou o aumento de estudos sobre plágio, fato que revela o aumento da preocupação com esse problema, coincidindo com o aumento do acesso à produção científica pelo meio digital e disponível na Internet. Dentre esses estudos, destaca-se àqueles que atribuem ao bibliotecário e aos educadores a responsabilidade na formação de estudantes comprometidos com a integridade científica. É importante que no Brasil também haja essa preocupação refletida na produção científica da área de Ciência da Informação. 
Artigo recebido em 08/04/20I6 e aceito para publicação em 04/05/20I8

\section{SCIENTIFIC PRODUCTION ON PLAGIARISM INDEXED IN LIBRARY DATABASE AND INFORMATION SCIENCE ABSTRACTS (LISA)}

ABSTRACT In this work we propose to reflect on the international scientific production related to the subject of plagiarism in the area of Information Science. This is a quantitative and exploratory research that analyzed scientific articles in the area of Information Science indexed in the Library and Information Science Abstracts (LISA) database. The corpus of analysis was composed of 35 I scientific articles on the subject. As a result, there is a concentration of articles on plagiarism from the 2000s; the main topics covered in the publications refer to copyright; the keyword plagiarism is mainly associated with the themes 'ethic', 'detection-tion', 'user training' and 'information literacy'. The most prominent journal is the Journal of Information Ethics (UTE) and the predominant language is English. The international scientific production of Information Science on plagiarism turns to several questions, highlighting studies that attribute to the librarian and educators the responsibility in the formation of students committed to scientific integration. It is important that in Brazil there is also this concern in the scientific production of the area of Information Science.

Keywords: $\quad$ Plagiarism; Scientific Integrity; Information Science; Bibliometric Indicators.

\section{REFERÊNCIAS}

ANDERSON, A. J. How do you manage: a lesson in plagiarism 101. Library Journal, v. 119, n. 10, p. 80-82, jun. 1994.

ASSOCIATION OF COLLEGE AND RESEARCH LIBRARIES. Information Literacy Competency Standards for Higher Education. 2000. Disponível em: <http://www.ala.org/acrl/ standards/informationliteracycompetency>. Acesso em: 03 maio 2016.

AUER, N. J.; KRUPAR, E. M. Mouse click plagiarism: the role of technology in plagiarism and the librarian's role in combating it. Library Trends, v. 49, n. 3, p.415-432, jan. 2001.

BADKE, W. Give plagiarism the weight it deserves. Online, v. 31, n. 5, p. 58-60, apr. 2007.

BARRON-CEDENO, A.; GUPTA, P.; ROSSO, P. Methods for cross-language plagiarism detection. Knowledge-Based Systems, n. 50, p. 211-217, sep. 2013.

BELLUZZO, R. C. B. O uso de mapas conceituais e mentais como tecnologia de apoio à gestão da informação e da comunicação: uma área interdisciplinar da competência em informação. Revista Brasileira de Biblioteconomia e Documentação, São Paulo, v. 2, n. 2, p. 78-89, dez. 2006.

BOMBARO, C. Using audience response technology to teach academic integrity: "the seven deadly sins of plagiarism". Reference Services Review, v. 35, n. 2, p. 296-309, 2007.

BRINE, A.; STUBBINGS, R. Plagiarism and the role of the library. Library + Information Update, v. 2, n. 12, p. 42-44, dec. 2003.

CAMERON, C.; ZHAO, H.; MCHUGH, M. K. Perspective: Publication ethics and the emerging scientific workforce: Understanding "plagiarism" in a global context. Academic Medicine, v. 87, p. 51-54, 2012.

DAMES, K. M. Understanding plagiarism and how it differs from copyright infringement. Computers in Libraries, v. 27, n. 6, p. 24-27, jun. 2007.

DAVARPANAH; M. R.; ASLEKIA, S. A scientometric analysis of international LIS journals: productivity and characteristics. Scientometrics, Dordrecht, v. 77, n. 1, p. 21-39, 2008. 
DELGADO, E.; RUIZ-PÉREZ, R.; JIMÉNEZCONTRERAS, E. La edición de revistas científicas. Directrices, criterios y modelos de evaluación. Madrid: FECYT, 2006. Disponível em: <http://www.fecyt.es/fecyt/ docs/ tmp/550433876.pdf>. Acesso em: 08 jun. 2016.

DUDZIAK, E. A. A information literacy e o papel educacional das bibliotecas. 2001. 187f. Dissertação (Mestrado em Comunicação) - Escola de Comunicação e Artes da Universidade de São Paulo, São Paulo, 2001.

DUGGAN, FIONA. The Plagiarism Advisory Service: promoting best practice in dealing with plagiarism. Library and Information Research News, v. 27, n. 86, p. 37-42, summer, 2003.

DURIEUX, V.; GEVENOIS, P. A. Bibliometric indicators: quality measurements of scientific publication. Radiology, v. 255, n. 2, may 2010.

ERCEGOVAC, ZORANA; RICHARDSON, JOHN V. Academic dishonesty, plagiarism included, in the digital age: a literature review. College and Research Libraries, v. 65, n. 4, p. 301-318, jul. 2004.

FISHER, B.; MOULTON, L.; PICCOLI, R. Competências para bibliotecários especializados do século XXI. Disponível em: <http://www.sla.org/Professional/comp.html>. Acesso em: 20 jan. 2016.

GIBSON, N. S.; CHESTER-FANGMAN, C. The Librarian's Role in Combating Plagiarism. Reference Services Review, v. 39, n. 1, p. 132150, 2011.

GIPP, B.; MEUSCHKE, N.; BREITINGER, C. Citation-Based Plagiarism Detection: Practicability on a Large-Scale Scientific Corpus. Journal of the Association for Information Science and Technology, v. 65, n. 8, p.1527-1540, aug. 2014.

GRESHAM, K. Preventing plagiarism on the Internet: teaching library researchers how and why to cite electronic sources. Colorado Libraries, v. 22, n. 2, p. 48-50, jul. 1996.
GUEDES, D. O.; GOMES FILHO, D. L. Percepção de plágio acadêmico entre estudantes do curso de odontologia. Revista Bioética, v. 23, n. 1, p. 139-148, 2015.

GUEDES, V.; BORSCHIVER, S. Bibliometria: uma ferramenta estatística para a gestão da informação e do conhecimento, em sistemas de informação, de comunicação e de avaliação científica e tecnológica. In: ENCONTRO NACIONAL DE CIÊNCIA DA INFORMAÇÃO, 6., 2005, Salvador - BA. Anais eletrônicos... Salvador: ICI/UFBA, 2005. Disponível em: <http:/ / dici.ibict.br/archive/00000508/01/ VaniaLSGuedes.pdf>. Acesso em: 26 set. 2007.

HABIBZADEH, F.; MARCOVITCH, H. Plagiarism: the emperor's new clothes. European Science Editing, v..37, n. 3, p.67-69, aug. 2011.

HAMILTON, D. Plagiarism: librarians help provide new solutions to an old problem. Searcher, v. 11, n. 4, p. 26-28, apr. 2003.

HANNAY, W M. Plagiarism on US campuses. Logos, v. 9, n. 2, p. 113-114, 1998.

HILL, J. D.; PAGE, E. F. An Empirical Research Study of the Efficacy of Two Plagiarism-Detection Applications. Journal of Web Librarianship, v. 3, n. 3, p. 169-181, jul. 2009.

JIMÉNEZ-CONTRERAS, E. Los métodos bibliométricos: estado de La cuestión y aplicaciones. In: Congreso Universitario de Ciencias de la Documentación, 1. Anais... Madrid, 2000.

KOZAK, E M. Towards a definition of plagiarism: The Bray/Oates controversy revisited. Journal of Information Ethics, v. 3, n. 1, p. 70-75, apr. 1994.

LE COADIC, Y. A Ciência da Informação. Brasília: Brinquet de Lemos, 1996.

LIBRARY AND INFORMATION SCIENCE ABSTRACTS. Sobre o LISA. [2016]. Disponível em: $\quad<$ http://search.proquest.com/lisa/ productfulldescdetail accountid $=14643>$. Acesso em: 06 jul. 2016. 
MADRAY, A. The Anatomy of a Plagiarism Initiative: One Library's Campus Collaboration. Public Services Quarterly, v. 4, n. 2, p. 111-125, 2008.

MUNDAVA, M.; CHAUDHURI, J. Understanding plagiarism: the role of librarians at the University of Tennessee in assisting students to practice fair use of information. College \& Research Libraries News, v. 68, n. 3, p. 170-173, mar. 2007.

OLIPHANT, T. Cyber-plagiarism: plagiarism in a digital world. Feliciter, v. 48, n. 2, p. 7880, 2002.

PADUA, G. C. C.; GUILHEM, D. Integridade científica e pesquisa em saúde no Brasil: revisão da literatura. Revista Bioética, v. 23, n. 1, p. 12438, 2015.

PHILLIPS, R. Plagiarism and theological education. Journal of Religious and Theological Information, v. 5, n. 2, p. 3-12, 2002.

PISTELLI, Z. La conservazione e la disponibilita delle tesi di laurea in un dipartimento universitário: the preservation and availability of degree theses in a university department. Bibliotecario, v.11/12, mar. 1987.

PRICE, A. R. Federal actions against plagiarism in research. Journal of Information Ethics, v. 5, n. 1, p. 34-51, apr. 1996.

RAHMAN, M.; FUKUI, T. A decline in the U.S. share of research articles. The New England Journal of Medicine, v. 347, n. 15, p. 1211-1212, 2002.

REES, H. C. The legal aspects of electronic information access. OFHE Bulletin, v. 82, p. 5-6, oct. 1997.

REYMAN, J. Rethinking plagiarism for technical communication. Technical Communication, v. 55, n. 1, p. 61-67, 2008.

RIGGAN, W. Plagiarism and reviewer/editor responsibility. Journal of Information Ethics, v. 6, n. 1, p. 34-38, apr. 1997.
SANTOS, L. H. L. Sobre a integridade ética da pesquisa. Boas práticas científicas. Fundação de Amparo à Pesquisa do Estado de São Paulo, 2011. Disponível em: <http://www.fapesp.br/6566>. Acesso em: 13 abr. 2016.

SEPULVEDA, O.; SEPULVEDA, O. A. Responsabilidad profesional en la produccion de la literature bibliotecologica. Professional responsibility and the production of library science literature. Arboleda Revista AIBDA, v. 2, n. 1, jan. 1981.

SILVA, M. R.; HAYASHI, M. C. P. I. Neobibliometria no contexto do neodocumentalismo. In: CRIPPA, G.; MOSTAFA, S. P. (Orgs). Ciência da informação e documentação. Campinas: Alínea, 2011. p. 71-84.

STEBELMAN, S. Cybercheating: dishonesty goes digital. American Libraries, v. 29, n. 8, p. 48-50, sep. 1998.

STORER, N. The social system of science. New York: Holt, Hinehardt and Winston, 1966.

TARGINO, M. G. A região geográfica como fator interveniente na produção de artigos de periódicos científicos. In: MUELLER, S. P. M.; PASSOS, E. J. L. (Orgs.). Comunicação científica. Brasília: Fundação Universidade de Brasília, 2000. p. 51-72.

VELHO, L. Ciências, publicações e avaliação. In: HOFFMANN, W. A. M.; FURNIVAL, A. C. M. Olhar: Ciência, Tecnologia e Sociedade. São Carlos: Pedro \& João Editores: CECH-UFSCar, 2008. p. 9-19.

WALTER, N. Only blockheads write for money. Library Association Record, v. 78, n. 4, apr. 1976.

ZIMERMAN, M. Plagiarism and international students in academic libraries. New Library World, v. 113, n. 5/6, p. 290-299, 2012. 